\title{
Surface activities of a lipid-analogue room temperature ionic liquid and its effects on phospholipid membrane
}

\author{
S. Mitra ${ }^{1}$, R. Das 2 , A. Singh ${ }^{3}$, M. K. Mukhopadhyay ${ }^{3}$, G. Roy ${ }^{2}$ and S. K. Ghosh ${ }^{1 *}$ \\ ${ }_{1}^{1}$ Department of Physics, School of Natural Sciences, Shiv Nadar University, NH 92, Tehsil Dadri, \\ G. B. Nagar, Uttar Pradesh 201314, India \\ ${ }^{2}$ Department of Chemistry, School of Natural Sciences, Shiv Nadar University, NH 92, Tehsil Dadri, \\ G. B. Nagar, Uttar Pradesh 201314, India \\ ${ }^{3}$ Surface Physics and Material Science Division, Saha Institute of Nuclear Physics, AF Block, \\ Bidhannagar, Kolkata 700064, India \\ *Corresponding author e-mail: sajal.ghosh@snu.edu.in
}

\section{Outlines:}

(1) Figure S1: Surface pressure-area isotherm of DPPC.

(2) Figure S2: XRR profile of bare Si substrate after dipping the substrate in water and dried in air (red) and in vacuum (black).

(3) Figure S3: AFM images obtained from (A) pristine DPPC, (B) pristine lipid analogue (LA) molecules and (C) DPPC monolayer mixed with $20 \mathrm{~mol} \%$ of LA. The monolayers are deposited at the sub-phase temperature of $25^{\circ} \mathrm{C}$ on the hydrophilic Si substrate at a surface pressure of 20 $\mathrm{mN} / \mathrm{m}$. 


\section{(1) Surface pressure - area isotherm of DPPC monolayer}

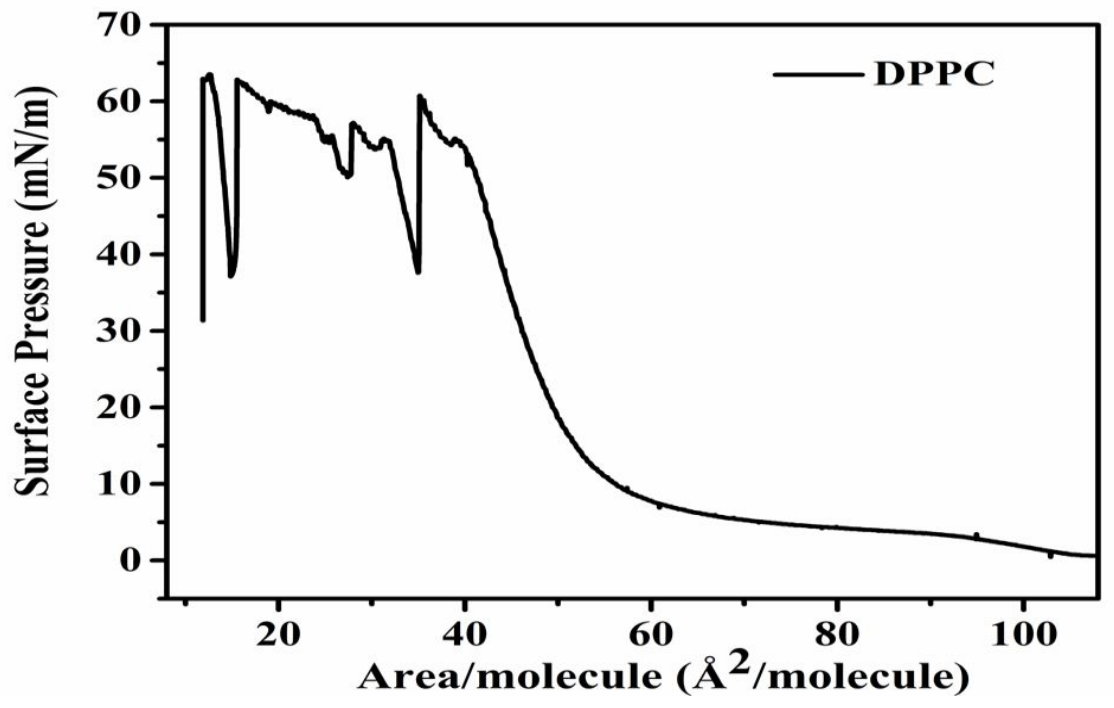

Figure S1. Surface pressure - area isotherm of DPPC monolayer at air-water interface measured at $25^{\circ} \mathrm{C}$. The abrupt drop of pressure at around $55 \mathrm{mN} / \mathrm{m}$ represents the constant area collapse of the monolayer that forms a lipid bilayer. The subsequent sudden drops of pressure signifies the formation of multilayer.

\section{(2) X-ray reflectivity profile of bare Si substrate:}

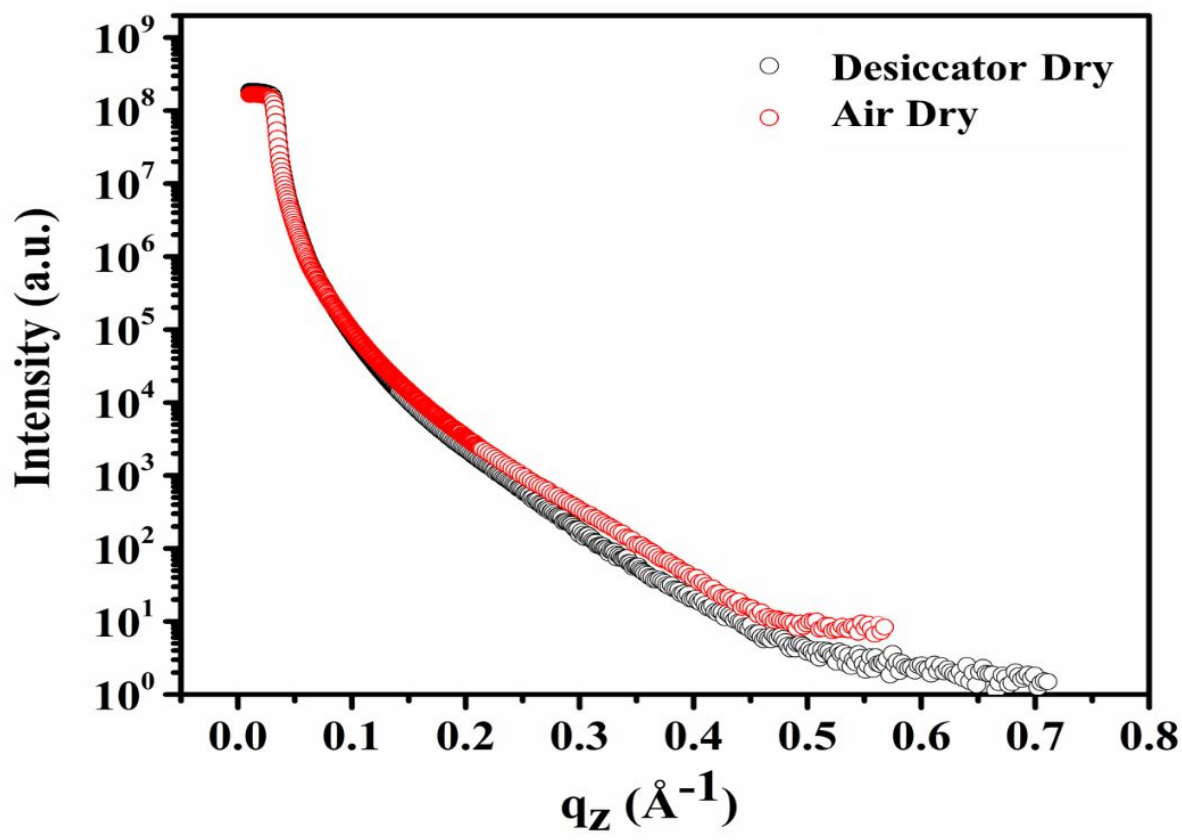

Figure S2. X-ray reflectivity (XRR) profile of bare hydrophilic Si substrate after dipping the substrate in water at $25^{\circ} \mathrm{C}$. The substrate was dried in air (red) and in a vacuum desiccator (black). No Kiessing fringes are observed in the XRR profile. Hence, there is no signature of the hydration layer observed. 

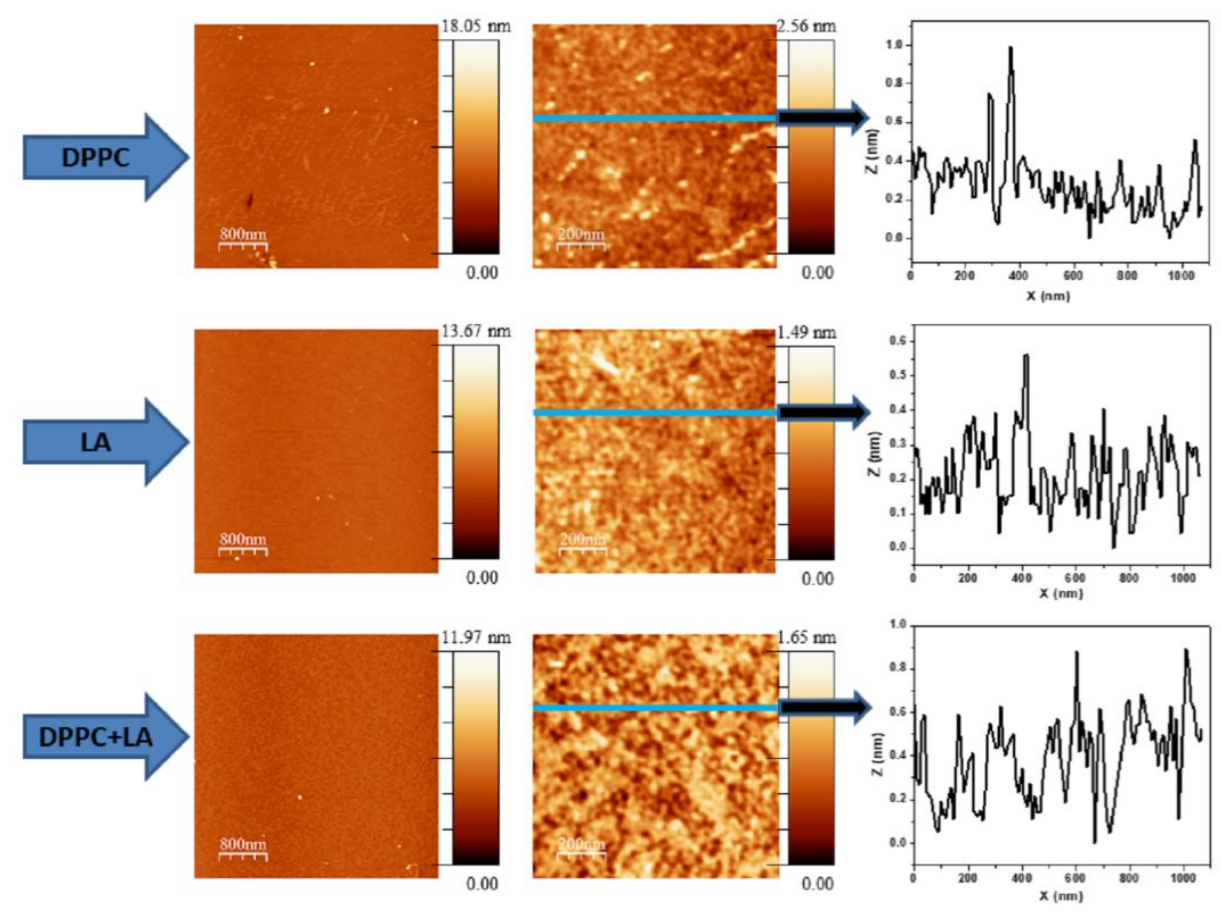

Figure S3. AFM images obtained from (A) pristine DPPC, (B) pristine lipid analogue (LA) molecules and (C) the DPPC monolayer mixed with $20 \mathrm{~mol} \%$ of LA molecules. The monolayers are deposited at the sub-phase temperature of $25^{\circ} \mathrm{C}$ on the hydrophilic Si substrate at a surface pressure of $20 \mathrm{mN} / \mathrm{m}$. NanoScope IV MMAFM, Veeco instrument was used for the study in tapping mode. The deposited monolayers were kept in a vacuum desiccator overnight before the measurement. The images show good coverage and uniform distribution of the layers on $\mathrm{Si}$ substrate. 